\title{
Variability of the counterpart to the gamma-ray blazar GT0106+613
}

\author{
Pedro L. Luque-Escamilla ${ }^{1,4}$, J. Martí ${ }^{2,4}$, E. Ramírez-Valenzuela ${ }^{3}$, \\ A. J. Muñoz-Arjonilla ${ }^{4}$ and J. M. Paredes ${ }^{5}$ \\ ${ }^{1}$ Dept. Ingeniería Mecánica y Minera, Universidad de Jaén, \\ E.P.S. Jaén, Campus Las Lagunillas, s/n, 23700, Jaén, Spain \\ email: peter@ujaen.es \\ ${ }^{2}$ Dept. Fsica, Universidad de Jaén, \\ E.P.S. Jaén, Campus Las Lagunillas, s/n, 23700, Jaén, Spain \\ email: jmarti@ujaen.es \\ ${ }^{3}$ Instituto de Astrofísica de Andalucía, CSIC, Avda. Vía Láctea \\ ${ }^{4}$ Grupo de Investigación FAEG, Univ. de Jaén, Campus Las Lagunillas s/n, 23071 Jaén, Spain \\ email: ajmunoz@ujaen.es \\ ${ }^{5}$ Departament dAstronomia i Meteorologia, Institut de Ciències del Cosmos (ICC), Universitat \\ de Barcelona (IEEC-UB), Martí i Franquès 1, E-08028, Barcelona, Spain. \\ email: jmparedes@ub.edu
}

\begin{abstract}
We present the optical/infrared counterpart to GT0106+613, a transient gamma-ray source believed to be a blazar. Long-term differential photometry and satellite information was used to confirm the variability in optical/infrared wavelengths, correlated with gamma-ray flares from the source. An intense optical flare with no counterpart in gamma-rays is also remarkable.
\end{abstract}

Keywords. gamma rays: observations, quasars: individual (J0109+6134)

\section{Introduction}

GT0106+613 is a variable radio source (Taylor \& Gregory (1983)). It has been detected as a flaring gamma and X-ray source by Fermi, AGILE and Swift (Vanderbroucke \& Hill (2010); Sabatini et al. (2010)). A faint optical IPHAS counterpart has been proposed (IPHAS J010946.33+613330.5; Knigge et al. (2010)), which revealed a blazar-like spectrum with redshift $z=0.7$ (Vandenbroucke et al. (2010)). Although a blazar could explain the gamma-ray emission, there exists no correlated optical/gamma variability to assure the physical relation between the different sources. In this work we summarize the results of a optical and infrared observations of the IPHAS candidate that provides strong evidence of variability in weekly time scales or even shorter, correlated with gamma flaring, which gives confidence to the proposed identification.

\section{Search strategy}

A photometric monitoring of the GT0106+613 field was carried out from August 2010 to January 2011 with the $2.0 \mathrm{~m}$ robotic Liverpool Telescope (LT) at the Roque de los Muchachos in Spain through B, V, r and $i$ filters. Only in this last filter the source was bright enough to be detected, exhibiting clear variability (see right part of Fig. 1). Its light curve may be seen in the left of Fig. 1 (upper panel) together with Fermi Large Area Telescope (LAT) gamma-ray ligh curve (middle panel) and the comparison star behaviour (lower panel; showing no variability). An optical flare with no correlation with 

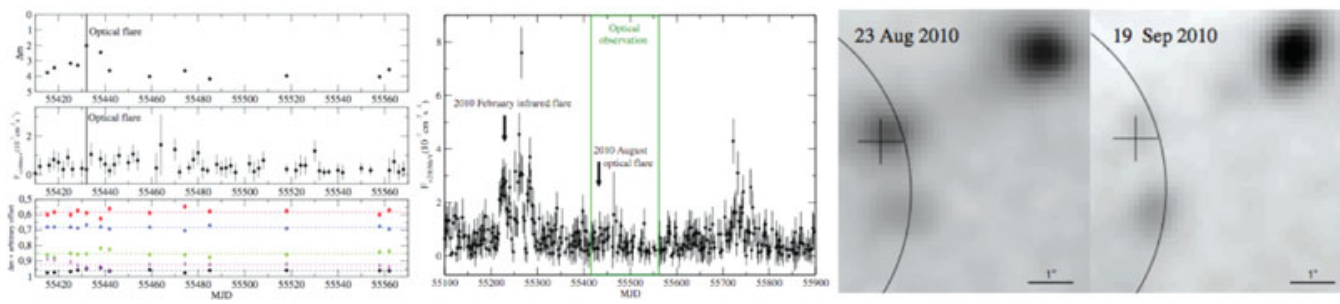

Figure 1. Left. Differential lightcurve for GT0106+613 in $i$-band (up) and Fermi-LAT for energies above $200 \mathrm{MeV}$ (middle). Comparison stars were stable (down). Center.Fermi-LAT light curve for GT0106+613. Flares and observation epochs are shown. Right Source variability observed with LT, $i$-filter. Cross and error circle are Chandra and Swift X-ray positions.

gamma-ray was detected. As may be seen in the middle of Fig. 1, an infrared flare was detected by Wide-field Infrared Survey Explorer (WISE) in February 2012 well correlated with a high-energy flare.

\section{Discussion}

The IPHAS source has an obvious infrared WISE counterpart with significative variability (almost a half of magnitude at 4.6 and $12 \mu \mathrm{m}$ ) correlated with gamma-rays. Our optical lightcurve shows weekly variability of $1.7 \mathrm{mag}$ or even shorter that seems not to be correlated with any gamma-ray flare (see Fig. 1), and resembles the behaviour of the well-studied case of PKS 0208-512 (Chatterjee et al. (2013)). This lack of correlation may be due to the nature of the optical synchrotron emission (magnitude/direction changes in the magnetic field while relativistic electrons and seed photons for Compton scattering responsible for gamma-ray emission remain constant), or to the location of the outburst along the relativistic jet flow (if it occurs close to the central black hole the optical emission dominates over the high energy one (Katarzynski \& Ghisellini (2007)).

\section{Conclusions}

A far infrared counterpart WISE J010946.32+613330.4 to the flaring gamma-ray blazar GT0106+61 is presented. Their photometric variability correlated with the high-energy emission observed by Fermi and the coincidence with the previously reported radio and optical (IPHAS) counterparts strongly support their physical connection. We also report for the first time an optical lightcurve of this IPHAS counterpart, revealing clear evidence of variability in week time scales. A remarkable $i$-band flare without correlation with gamma-ray flux increase has been observed. This unusual aspect of blazar behaviour has been previously detected in PKS 0208-512, and its interpretation is still under debate.

\section{References}

Chatterjee, R, Fossati, G., Urry, C. M., et al. 2013, ApJ, 763, L11

Katarzynski, K. \& Ghisellini, G. 2007, A\& $A$, 463, 529

Knigge, C., Steeghs, D., Gaensicke, B., et al. 2010, ATel, 2429

Sabatini, S., Tavani, M., Bulgarelli, A., et al. 2010, ATel, 2416

Taylor, A.R. \& Gregory, P.C. 1983, AJ, 88, 1784

Vandenbroucke, J. \& Hill, A. B., on behalf of the Fermi LAT Collaboration 2010, ATel, 2414

Vandenbroucke, J., Buehler, R., Ajello, M., et al. 2010, ApJ, 718, L166 